\title{
Attention and memory cues in concept learning as a function of task complexity and age*
}

\author{
VLADIMIR PISHKIN and ELIZABETH A. RASMUSSEN \\ VA Hospital and University of Oklahoma, College of Medicine, Oklahoma City, Oklahoma 73104
}

\begin{abstract}
The effects of attention and memory cues on concept learning performance were investigated as a function of task complexity and grade level of Ss. It was found that: (1) the attention and memory cues were closely interrelated in their effects upon performance with both significantly improving performance at all but the lowest levels of complexity; (2) both types of cues considerably lessened the effect of stimulus complexity upon performance; and (3) the attention cue elicited the best performance from $S s$ in the first through ninth grades, while the memory cue elicited the best performance from 4-year-old and kindergarten Ss.
\end{abstract}

Attention and memory have been stressed as processes underlying some of the basic changes which occur within cognitive development. The importance of attentional factors in children's learning is pointed out by recent studies of Zeaman and House (1963), Suchman and Trabasso (1966), and also by work with the orienting response (Sokolov, 1963; Zaporozhets, 1960). The role of memory functions in cognitive growth is stressed by Bruner (1964) with his considerations of changing operational memory systems within the child and by Wohlwill (1962) who emphasizes decreasing dependence on information within the immediate stimulus field as a function of age. A major purpose of the present study was to explore the influence of attention and memory cues on cognitive performance with Ss from preschool through junior high school age.

In concept identification research, specific experimental manipulations have been operationally linked with the processes of attention and memory. Techniques to establish cue or dimensional preferences through pretesting, pretraining, or the use of emphasizers within the task have been connected with attentional requirements of conceptual tasks. These manipulations have been found to both improve and hinder performance, depending on whether the emphasis has been placed on the saliency of the relevant or the irrelevant dimensions of the task (Rasmussen \& Archer, 1961; Archer, 1962; Trabasso, 1963; Suchman \& Trabasso, 1966; Stephens, 1968). Past stimulus availability has been associated with the memory requirements of concept identification tasks and has been found to improve performance for fourth-grade through college Ss (Cahill \& Hovland, 1960; Bourne, Goldstein, \& Link, 1964; Pishkin \& Wolfgang, 1965; Pishkin, Wolfgang, \& Rasmussen, 1967).

These manipulations associated with attention and memory processes have generally been studied

*Thanks are given to the staff and students of Casady School, Oklahoma City, Oklahoma, for their cooperation and participation. separately. In order to obtain a clearer picture of the effects of these manipulations upon performance, they were compared over several levels of task complexity as well as with several age groups in the present study.

The attention and memory cues used in this experiment followed manipulations that have been utilized in other concept learning experiments. The memory aid used was the presence of a specific past correct instance within each sorting category as used by Pishkin and Wolfgang (1965) and Pishkin, Wolfgang, and Rasmussen (1967). The attention cue followed that used in the rule learning task of Haygood and Bourne (1965) in which S's attention was focused on the levels of the relevant dimension by visual presentation of these stimulus attributes to the $\mathrm{S}$ prior to and during the task. A condition in which no cues were available was used to provide a control condition comparable to the typical concept learning situation.

\section{METHOD}

\section{Subjects}

The Ss for the study were 180 males from the 4-year-old preschool through the ninth-grade classes at the Casady School in Oklahoma City, Oklahoma. The Ss were divided into four grade-level groups: the first group consisted of 45 students from the preschool (4year-olds) and kindergarten classes (Group P); the second group was composed of 45 students from Grades 1 , 2 , and 3 (Group 2); the third group consisted of 45 students from Grades 4, 5, and 6 (Group 5); and the fourth group was composed of students from Grades 7,8, and 9 (Group 8). With the restriction that an equal number of $S$ s from each grade level be assigned to each complexity and cue availability condition, the Ss were randomly assigned to the design cells with 5 Ss per cell.

\section{Design}

This was a 4 by 3 by 3 factorial design. The variables were: the four grade-level groups, three conditions of cue availability, and three levels of stimulus complexity within each grade group. In addition, two problems were used to aid in minimizing the effect of spread of information among the Ss. Thethree conditions of cue availability were: (1) last specific correct 
Table 1

Stimulus Complexity Levels Within Each Grade Level

\begin{tabular}{lccc}
\hline & \multicolumn{3}{c}{ Pooled Complexity Level* } \\
\cline { 2 - 4 } Grade Level & Low & Middle & High \\
\hline Preschool-Kindergarten & 1 & 2 & 3 \\
Grades 1, 2, and 3 & 2 & 3 & 4 \\
Grades 4, 5, and 6 & 3 & 4 & 5 \\
Grades 7, 8, and 9 & 4 & 5 & 6 \\
Median Complexity & 2.5 & 3.5 & 4.5 \\
\hline
\end{tabular}

*Number of irrelevant dimensions.

instances left exposed within each category (memory aid condition); (2) the levels of the relevant dimension shown to $S$ and left exposed throughout the task (attention cue condition); and (3) no cues available for $S$ (no cues condition). Within each grade-level group, problems of three stimulus complexity levels, labeled low, middle, and high, were presented. Because of the grade range within each group and the trends of improvement in concept learning with age (e.g., Osler \& Kofsky, 1965), the specific stimulus complexity levels were not identical for the grade groups but were overlapped in order to provide a range of problem difficulty in keeping with the S's age-related attributes. Table 1 shows how the specific stimulus complexity levels varied with grade grouping. The two problems used were shape as the relevant dimension and color as the relevant dimension; two or three Ss within each design cell worked on each problem type.

\section{Procedure}

S's task was to sort geometric patterns on white $3 \times 5$ in. cards into two slots of a wood sorting tray placed in front of him. A separate deck of 96 cards was used for each stimulus complexity level. The cards within each deck were arranged in a random order, with the restriction that the same pattern could not immediately follow itself. All the stimulus dimensions were binary. The relevant dimension was shape (square and triangle) for one-half of the Ss and color (red and blue) for the other half of the Ss. The other five dimensions, added progressively as the complexity level increased, were: size $(2.5 \mathrm{~cm}$ and $1.25 \mathrm{~cm}$ in vertical height), number (one or two patterns on the card), horizontal position (pattern on the left or right side of the card with the center of the pattern being $3.75 \mathrm{~cm}$ from the corresponding edge), vertical position (pattern on the top or bottom of the card with the center of the pattern being $2.5 \mathrm{~cm}$ from the corresponding edge), and orientation (pattern tilted or in its normal position -in the tilted position the squares were rotated $45 \mathrm{deg}$, thus appearing as diamonds, and the triangles were rotated $180 \mathrm{deg}$, thus appearing as inverted triangles).

The Ss sat at a table opposite E with the two-slot card-sorting tray in front of S. A card file containing the deck of cards was next to the tray. The Ss were told that their task was to sort the cards into the two slots and the $E$ would inform them whether they were right or wrong after each choice. The Ss were further instructed that when they were wrong, they were to place the card in the correct slot before continuing. Ss in the no cues condition were told to place the cards face down (pattern not showing) in the correct slot. Ss in the memory aid condition were told to place the cards face up in the correct slot; each new card placed in the slots covered the past cards so that only one past correct instance was visible within each category. In the attention cue condition, the Ss were instructed to place the cards face down in the correct slot; in addition, at the end of their instructions, these Ss were shown cards depicting the levels of the relevant dimension (for shape relevant, cards with a black outline of a square and a black outline of a triangle were presented; for color relevant, cards covered with red and blue construction paper were presented). The attention cue Ss were informed only that this was one of the ways in which the cards they would see would differ from each other. No verbal labels were given to these cards by $\mathrm{E}$. The Ss pe.formed on the task until they had made 16 consecutive correct responses or for a total of 96 trials. Ss worked on the task at their own pace.

\section{RESULTS AND DISCUSSION}

The dependent variable was the number of errors made prior to criterion. Due to the presence of heterogeneity of variance (Hartley $F_{\max }$ test) produced by the positive skewness of error score distributions within a number of cells, a $\log (x+1)$ transformation of the error scores was made (Winer, 1962, p. 221). This transformation was effective in removing the heterogeneity of variance. All subsequent analyses are based on these log error scores.

In order to determine whether the problem type (i.e., shape relevant or color relevant) was a significant source of variance, $t$ tests were performed on the log error scores of the two problems within each grade group. The t-test results were: Group P, $t=0.22, \mathrm{df}=43$; Group 2, $\mathrm{t}$ $=0.79, \mathrm{df}=43 ;$ Group 5, $\mathrm{t}=0.11, \mathrm{df}=43 ;$ and Group 8, $t=0.63$, $d f=43$. Since none of the ts approached significance, in all subsequent analyses the two problems were pooled.

A repeated measures analysis of variance on blocks of 12 trials was performed. All of the main effects and several of the interactions were significant.

Even with the increasing complexity accompanying the increase in grade levels (see Table 1), the mean log errors decreased significantly with increasing age $(F=$ $3.61, \mathrm{df}=3 / 144, \mathrm{p}<.025)$. This trend was linear and the slope reliably different from zero, as shown by a significant linear component $(F=9.86, d f=1 / 144$, $\mathrm{p}<.01$ ). Application of the Tukey test, Type B (Winer, 1962 , p. 87) indicated that all comparisons between the groups were significant at $p<.01$, with the exception of Groups 2 and 5 which were not significantly different. The increasing efficiency of performance with S's increased grade level supports other work showing improvement in conceptual abilities with age (e.g., Osler \& Kofsky, 1965). However, this result also indicates that problem difficulty was not equated among the age groups, even with the overlapping used in the present study, and thus prevents exact comparisons among the age groups.

The main effect of complexity was significant $(F=$ $5.76, \mathrm{df}=2 / 144, \mathrm{p}<.01$ ) with mean $\log$ errors increasing with the complexity levels; this trend was linear. Subsequent testing (Tukey test, Type B) showed that all three levels of complexity were significantly different from each other at $\mathrm{p}<.01$. This effect follows the generally found trends of stimulus complexity (Archer, Bourne, \& Brown, 1955; Pishkin, 1960). The cue availability main effect was also significant $(F=$ 7.84 , df $=2 / 144, p<.01$ ), with the error rate being reliably reduced by the introduction of the memory aid $(\bar{x}=2.28)$ at the .01 level (Tukey test, Type B). The 
overall difference between the memory aid and the attention cue was not significant.

Due to the presence of a significant Complexity by Cue Availability interaction $(F=2.57$, df $=4 / 144$, $\mathrm{p}<.05$ ), shown in Fig. 1 , simple effects analyses of variance were performed on these two variables. Cue availability was not a significant source of varianca at the low complexity level. For the middle complexity level, no cues elicited significantly poorer performance than the memory aid and the attention cue (middle complexity, $\mathrm{F}=6.44, \mathrm{df}=2 / 144, \mathrm{p}<.005$ and Tukey's $B$ test at $p<.01$ for no cue-memory aid and no cue-attention cue differences); the difference between the memory aid and attention cue was not significant. At the high complexity level, all three cue availability conditions were significantly different from each other, with the most inefficient performance occurring in the no cues condition and the best performance occurring with the attention cue (high complexity, $\mathrm{F}=6.48, \mathrm{df}=$ $2 / 144, p<.005$, Tukey's $B$ test at $p<.05$ for the memory aid-attention cue difference).

The simple effects analysis of variance on complexity indicated that complexity was a significant variable only for the no cues condition $(F=8.86, \mathrm{df}=2 / 144$, $\mathrm{p}<.005)$. For the memory aid and attention cue, complexity was not a significant source of variation ( $F$ $=1.64$ and $0.28, \mathrm{df}=2 / 144$ ), although with the memory aid the increase in errors from the middle to high complexity levels was significant (Tukey's B test at $\mathrm{p}<.01)$. Thus, it is clear that the memory aid and attention cue conditions have the effect of reducing problem difficulty. To understand these differences, it might be helpful to examine the types of information contained in the cue availability conditions. The memory aid provides $S$ with immediate stimulus field information and the strict association between the stimulus and correct response category which the attention cue does not provide. The attention cue, on the other hand, contains only information related to the

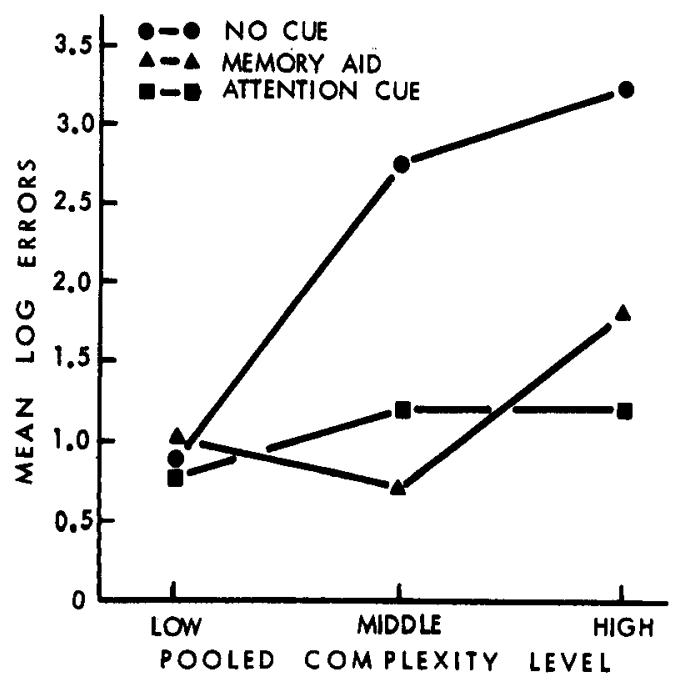

Fig. 1. Mean log errors for the three cue availability conditions as a function of pooled complexity level.

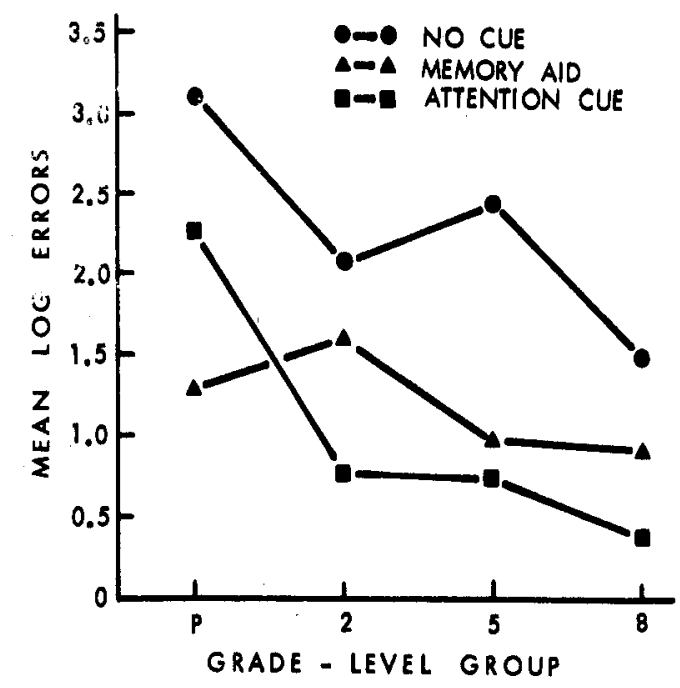

Fig. 2. Mean log errors for the three cue availability conditions as a function of grade level (Group $P=$ preschool and kindergarten; Group 2 = Grades 1, 2, and 3; Group $5=$ Grades, 4, 5, and 6; and Group $8=$ Grades 7, 8, and 9).

relevant stimulus dimension, while the memory aid contains both relevant and irrelevant stimulus information. While the results indicate that the information provided by both types of cues is effective in improving performance, only for higher stimulus complexity levels does the nonconfounded (i.e., only relevant stimulus information, not relevant and irrelevant stimuli information) attention cue provide an advantage for the $S$.

The Grade by Cue Availability interaction was not significant $(F=.87, \mathrm{df}=6 / 144, \mathrm{p}<.10)$; however, a major interest of this study was the effectiveness of the cue conditions for the different grade levels. To minimize the possibility that Type I errors would occur, the differences between the cue availability means for each group were tested by the Tukey test, Type B (Petrinovich \& Hardyck, 1969). These means are shown in Fig. 2. For Group P, all three cue availability conditions produced significantly different means, with the memory aid eliciting a superior performance level compared to both the attention cue and no cues conditions $(p<.01)$; the attention cue produced significantly fewer errors than did the no cues $(p<.05)$. For Group 2, the attention cue demonstrated the best performance compared to both the no cues $(\mathrm{p}<.01)$ and the memory aid conditions $(\mathrm{p}<.05)$; no reliable differences occurred between the no cues and memory aid groups. While both the memory aid and attention cue produced better performance than the no cues $(p<.01)$ for Group 5, with the best performance occurring with the attention cue, the differences between the memory aid and attention cue were not significant. For Group 8, the attention cue condition resulted in better performance than the no cues condition $(p<.01)$; however, the differences between the no cues and memory aid and the memory aid and attention cue were not significant. In terms of this 
analysis, the memory aid produced superior performance for Group P; the other groups learned better in the attention cue condition. The difference between the memory aid and attention cue was significant only for the two lower grade-level groups, Group P and Group 2 $(p<.01$ and $p<.05$, respectively). These results, however, can only be termed as suggestive and in need of further replication because of the overall nonsignificance of the Grade by Cue Availability interaction and the significant grade main effect indicating that the complexity levels were not psychologically equivalent for the grade groups. The tentative result indicating the superiority of the memory aid for the preschool Ss supports recent theorizing in cognitive development. For example, Bruner (1964) and Wohlwill (1962) have emphasized the role of the perceptual qualities of, and dependence on, the immediate stimulus field in cognition of the young child. The memory aid does, in fact, provide immediate stimulus information to the $S$ which the attention cue does not.

The main effect of blocks (based on units of 12 successive trials) was highly reliable as expected with mean $\log$ errors decreasing as a function of trials ( $\mathrm{F}=$ 130.33, $\mathrm{df}=7 / 1008, \mathrm{p}<.001$ ). The largest decrease in mean $\log$ errors occurred between the first and second blocks, with the decrease then becoming more gradual. This change in rate of error decrease is reflected in the significance of the linear, quadratic, and cubic components of this main effect.

The significant Blocks by Grade interaction $(F=1.79$, $\mathrm{df}=21 / 1008, \mathrm{p}<.025)$ indicates that the decrease in error rate across trials was progressively more rapid with increased grade level. This differential rate of error decrease is also reflected in the significant quadratic component of the trend. The Blocks by Cue Availability interaction $(F=3.05, \mathrm{df}=14 / 1008, \mathrm{p}<.01)$ showed that the attention cue, while producing a significantly lower error rate on overall performance, also elicited improved performance, compared to the no cues and attention cue, on the first block. However, the memory aid produced performance levels similar to the no cues on the first block, then rapidly improved performance (reaching the attention cue level) by the second block.

It is clear that the Ss of all age groups studied in this experiment were able to utilize effectively the attention aid and memory cue to facilitate their performance. The influence of both types of cues (memory aid and attention cue) was to improve performance significantly, compared to a condition of no cues available, at all but the lowest levels of complexity. The differential effectiveness of these cues was significant: (1) in the early stages of performance (with attention cue eliciting better performance than memory aid); (2) at the highest complexity levels (with attention cue eliciting better performance than memory aid); and (3) for the youngest group of Ss (for the preschool Ss, memory aid elicited significantly better performance than attention cue; for the older Ss, attention cue produced better performance than memory aid, although the attention cue-memory aid difference was not significant for the two oldest groups).

\section{REFERENCES}

Archer, E. J. Concept identification as a function of obviousness of relevant and irrelevant information. Journal of Experimental Psychology, 1962, 63, 616-620.

Archer, E. J., Bourne, L. E., Jr., \& Brown, F. G. Concept identification as a function of irrelevant information and instructions. Journal of Experimental Psychology, 1955, 49, 153-164.

Bourne, L. E., Jr., Goldstein, S., \& Link, W. E. Concept learning as a function of availability of previously presented information. Journal of Experimental Psychology, 1964, 67, 439-448.

Bruner, J. S. The course of cognitive growth. American Psychologist, 1964, 19, 1-15.

Cahill, H. E., \& Hovland, C. I. The role of memory in the acquisition of concepts. Journal of Experimental Psychology, $1960,59,137-144$.

Haygood, R. C., \& Bourne, L. E., Jr. Attribute- and rule-learning aspects of conceptual behavior. Psychological Review, 1965, 72, 175-195.

Osler, S. F., \& Kofsky, E. Stimulus uncertainty as a variable in the development of conceptual ability. Journal of Experimental Child Psychology, 1965, 2, 264-279.

Petrinovitch, L. F., \& Hardyk, C. D. Error rates for multiple comparison methods: Some evidence concerning the frequency of erroneous conclusions. Psychological Bulletin, $1969,71,43-54$.

Pishkin, V. Effects of probability of misinformation and number of irrelevant dimensions upon concept identification. Journal of Experimental Psychology, 1960, 59, 371-378.

Pishkin, V., \& Wolfgang, A. Number and type of available instances in concept learning. Journal of Experimental Psychology, 1965, 69, 5-8.

Pishkin, V., Wolfgang, A., \& Rasmussen, E. .Age, sex, amount and type of memory information in concept learning. Journal of Experimental Psychology, 1967, 73, 121-124.

Rasmussen, E. A., \& Archer, E. J. Concept identification as function of language pretraining and task complexity. Journal of Experimental Psychology, 1961, 61, 437-441.

Sokolov, Y. N. Perception and the conditioned reflex. New York: Macmillan, 1963.

Stephens, W. C. Concept learning with three types of verbal labels. Psychonomic Science, 1968, 10, 225-226.

Suchman, R. G., \& Trabasso, T. Stimulus preference and cue function in young children's concept attainment. Journal of Experimental Child Psychology, 1966, 3, 188-198.

Trabasso, T. Stimulus emphasis and all-or-none learning of concept identification. Journal of Experimental Psychology, $1963,65,395-405$.

Winer, B. J. Statistical principles in experimental design. New York: McGraw-Hill, 1962.

Wohlwill, J. F. From perception to inference: A dimension of cognitive development. In W. Kessen and C. Kuhlman (Eds.), Thought in the young child. Monographs of the Society for Research in Child Development, 1962, 27(No. 12, Serial No. 83), 87-107.

Zaporozhets, A. V. Razvitie Proizvol' Nykh Dvizhenii. (The development of voluntary movements.) Moscow: Academy of Pedogogical Science, 1960.

Zeaman, E., \& House, B. J. The role of attention on retardate discrimination learning. In N. R. Ellis (Ed.), Handbook of mental deficiency. New York: McGraw-Hill, 1963. Pp. 159-223.

(Received for publication September 28, 1973; accepted October 22, 1973.) 